\title{
Secure Transmission With Artificial Noise Over Fading Channels: Achievable Rate and Optimal Power Allocation
}

\author{
Xiangyun Zhou, Student Member, IEEE, and Matthew R. McKay, Member, IEEE
}

\begin{abstract}
We consider the problem of secure communication with multiantenna transmission in fading channels. The transmitter simultaneously transmits an information-bearing signal to the intended receiver and artificial noise to the eavesdroppers. We obtain an analytical closed-form expression of an achievable secrecy rate and use it as the objective function to optimize the transmit power allocation between the information signal and the artificial noise. Our analytical and numerical results show that equal power allocation is a simple yet near-optimal strategy for the case of noncolluding eavesdroppers. When the number of colluding eavesdroppers increases, more power should be used to generate the artificial noise. We also provide an upper bound on the SNR, above which, the achievable secrecy rate is positive and shows that the bound is tight at low SNR. Furthermore, we consider the impact of imperfect channel state information (CSI) at both the transmitter and the receiver and find that it is wise to create more artificial noise to confuse the eavesdroppers than to increase the signal strength for the intended receiver if the CSI is not accurately obtained.
\end{abstract}

Index Terms-Artificial noise, channel estimation error, multiantenna transmission, power allocation, secrecy rate.

\section{INTRODUCTION}

$\mathbf{S}$ ECURITY IS A fundamental problem in wireless communications due to the broadcast nature of the wireless medium. Traditionally, secure communication is achieved by using cryptographic technologies such as encryption. On the other hand, studies from an information-theoretic viewpoint have found conditions for reliable secure communication without using secret keys. In the pioneering works on informationtheoretic security, Wyner introduced the wiretap channel model in which the eavesdropper's channel is a degraded version of the receiver's channel [1]. Csiszár and Körner considered a general nondegraded channel condition and studied the transmission of both a common message to two receivers and a

Manuscript received December 17, 2009; revised April 26, 2010 and June 30, 2010; accepted June 30, 2010. Date of publication July 26, 2010; date of current version October 20, 2010. This paper was presented in part at the International Conference on Signal Processing and Communication Systems, Omaha, NE, September 28-30, 2009. This work was supported by the Australian Research Council's Discovery Projects under Project DP0773898. The review of this paper was coordinated by Prof. Y. Zhang.

$\mathrm{X}$. Zhou is with the Research School of Information Sciences and Engineering, Australian National University, Canberra, ACT 0200, Australia (e-mail: xiangyun.zhou@anu.edu.au).

M. R. McKay is with the Department of Electronic and Computer Engineering, Hong Kong University of Science and Technology, Kowloon, Hong Kong (e-mail: eemckay@ust.hk).

Color versions of one or more of the figures in this paper are available online at http://ieeexplore.ieee.org.

Digital Object Identifier 10.1109/TVT.2010.2059057 confidential message to only one of them [2]. The results in these early works showed that a positive secrecy capacity can be achieved if the intended receiver has better channel than the eavesdropper.

Recently, information-theoretic security with multiantenna transmission has drawn a lot of attention. Many works have been devoted to analyzing the secrecy capacity with various antenna configurations and channel conditions, e.g., [3]-[6]. With multiple antennas at the transmitter, the optimal input structure (for Gaussian codes) that maximizes the secrecy rate of Gaussian channels was found to be in the form of beamforming transmission [3], [4]. The secrecy capacity of Gaussian channels with multiple antennas at both the transmitter and the receiver was obtained in [5] and [6]. One of the main assumptions in the aforementioned works is that the eavesdropper's channel is known at the transmitter. Clearly, this assumption is usually impractical, particularly for fading channels. The ergodic secrecy capacity with and without knowing the eavesdropper's channel was studied for fading channels in [7]-[11]. The authors in [9] studied a fading broadcast channel with confidential information intended only for one receiver and derived the optimal power allocation that minimizes the secrecy outage probability. The authors in [10] proposed an on-off power transmission with variable-rate-allocation scheme for singleantenna systems, which was shown to approach the optimal performance at asymptotically high SNR. The authors in [11] extended the ergodic secrecy capacity result to systems with multiple antennas and developed capacity bounds in the large antenna limit.

Furthermore, various physical-layer techniques were proposed to achieve secure communication, even if the receiver's channel is worse than the eavesdropper's channel. One of the main techniques is the use of interference or artificial noise to confuse the eavesdropper. With two base stations connected by a high-capacity backbone, one base station can simultaneously transmit an interfering signal to secure the uplink communication for the other base station [12], [13]. In the scenario where the transmitter has a helping interferer or a relay node, the secrecy level can also be increased by having the interferer [14] or relay [15] to send codewords independent of the source message at an appropriate rate. When multiple cooperative nodes are available to help the transmitter, the optimal weights of the signal transmitted from cooperative nodes, which maximize an achievable secrecy rate, were derived for both decode-and-forward [16] and amplify-and-forward [17] protocols. The use of interference for secrecy is also extended 
to multiple access and broadcast channels with user cooperation [18]-[20].

When multiple antennas are available at the transmitter, it is possible to simultaneously transmit both the informationbearing signal and the artificial noise to achieve secrecy in a fading environment [21]-[23]. The artificial noise is radiated isotropically to mask the transmission of the information signal to the intended receiver. In the design of this multiantenna technique, the transmit power allocation between the information signal and the artificial noise is an important parameter, which has not been investigated in [21] and [22]. A suboptimal power allocation strategy was considered in [23], which aims to meet a target signal-to-interference-and-noise ratio at the intended receiver to satisfy a quality of service requirement.

In this paper, we study the problem of secure communication in fading channels with a multiantenna transmitter capable of simultaneous transmission of both the information signal and the artificial noise. We derive a closed-form expression for an achievable secrecy rate in fading channels. The availability of a closed-form secrecy rate expression greatly reduces the complexity of obtaining the optimal power allocation between transmission of the information signal and the artificial noise. We also study the critical SNR above which the achievable secrecy rate is positive. This is an important problem in wideband communications in which a higher throughput is achieved by reducing the SNR per hertz while increasing the bandwidth [24]. Furthermore, perfect channel state information (CSI) at both the transmitter and the receiver is usually assumed in the existing studies on information-theoretic security. With this assumption, the artificial noise is accurately transmitted into the null space of the intended receiver's channel. When the CSI is not perfectly known at the transmitter, the artificial noise leaks into the receiver's channel. The effects of imperfect CSI on the achievable secrecy rate and the aforementioned design parameters are investigated.

The main contributions of this paper are the following.

1) In Section III, we derive analytical closed-form lower bounds on the ergodic secrecy capacity for both noncolluding and colluding eavesdroppers. These closedform expressions, which give achievable secrecy rates for secure communications with artificial noise, greatly reduce the complexity of system design and analysis and also allow analytical insights to be obtained.

2) In Section IV, we study the optimal power allocation between transmission of the information signal and the artificial noise. For the noncolluding eavesdropper case, the equal power allocation is shown to be a simple strategy that achieves nearly the same secrecy rate as the optimal power allocation. For the colluding eavesdropper case, more power should be used to transmit the artificial noise as the number of eavesdropper increases. Analytical results are obtained in the high SNR regime in both cases.

3) In Section V, we derive an upper bound on the critical SNR above which the achievable secrecy rate is positive. The bound is shown to be tight at low SNR and hence is useful in the design and analysis of wideband secure communications.
4) In Section VI, we derive an ergodic secrecy capacity lower bound taking into account channel estimation errors and investigate the effects of imperfect CSI on the optimal power allocation and the critical SNR for secure communication. In particular, we find that it is better to create more artificial noise for the eavesdroppers than to increase the signal strength for the intended receiver as the channel estimation error increases.

Throughout this paper, the following notations will be used: Boldface upper and lower cases denote matrices and vectors, respectively. $[\cdot]^{T}$ denotes the matrix transpose operation, $[\cdot]^{*}$ denotes the complex conjugate operation, and $[\cdot]^{\dagger}$ denotes the conjugate transpose operation. The notation $E\{\cdot\}$ denotes the mathematical expectation. $\|\cdot\|$ denotes the norm of a vector, and $|\cdot|$ denotes the determinant of a matrix.

\section{SySTEM MODEL}

We consider secure communication between a transmitter (Alice) and a receiver (Bob) in the presence of eavesdroppers (Eves). Alice has $N_{A}$ antennas $\left(N_{A}>1\right)$, and Bob has a single antenna. This scenario is representative, for example, of downlink transmission in cellular systems and wireless local area networks. In addition, each Eve is equipped with a single antenna. We consider two cases, namely, noncolluding and colluding eavesdroppers. In the former case, Eves individually overhears the communication between Alice and Bob without any centralized processing. Whereas in the latter case, there are $N_{E}$ Eves capable of jointly processing their received information. Therefore, the noncolluding case can be seen as a special colluding case where $N_{E}=1$. We assume that $N_{A}>N_{E}$, for which the reason will become clear in the next section. We also assume that Eves are passive; hence, they cannot transmit jamming signals. The received symbols at Bob and the multiple colluding Eves are, respectively, given by

$$
\begin{gathered}
y_{B}=\boldsymbol{h} \boldsymbol{x}+n \\
\boldsymbol{y}_{E}=\boldsymbol{G} \boldsymbol{x}+\boldsymbol{e}
\end{gathered}
$$

where $\boldsymbol{h}$ is a $1 \times N_{A}$ vector denoting the channel between Alice and Bob, and $G$ is an $N_{E} \times N_{A}$ matrix denoting the channel between Alice and multiple colluding Eves. The elements of $\boldsymbol{h}$ and $G$ are independent zero-mean complex Gaussian random variables. $n$ and $e$ are the additive white Gaussian noises at Bob and Eves, respectively. Without loss of generality, we normalize the variance of $n$ to unity. We assume that $\boldsymbol{h}$ is accurately estimated by Bob and is also known by Alice using a noiseless feedback link from Bob. ${ }^{1}$ Similar to [21], we assume that knowledge of both $\boldsymbol{h}$ and $\boldsymbol{G}$ is available at Eve, which makes the secrecy of communication independent of the secrecy of channel gains.

\footnotetext{
${ }^{1}$ A reliable feedback link could be achieved by using a low-rate transmission with appropriate quantization schemes. The design of a high-quality feedback link and the effect of noisy feedback are beyond the scope of this paper. However, we will investigate the effect of imperfect channel knowledge at Alice by considering channel estimation errors at Bob in Section VI.
} 
The key idea of guaranteeing secure communication using artificial noise proposed in [21] is outlined as follows: We let an $N_{A} \times N_{A}$ matrix $\boldsymbol{W}=\left[\begin{array}{ll}\boldsymbol{w}_{1} & \boldsymbol{W}_{2}\end{array}\right]$ be an orthonormal basis of $\mathbb{C}^{N_{A}}$, where $\boldsymbol{w}_{1}=\boldsymbol{h}^{\dagger} /\|\boldsymbol{h}\|$. The $N_{A} \times 1$ transmitted symbol vector at Alice is given by $\boldsymbol{x}=\boldsymbol{w}_{1} u+\boldsymbol{W}_{2} \boldsymbol{v}$, where the variance of $u$ is $\sigma_{u}^{2}$, and the $N_{A}-1$ elements of $\boldsymbol{v}$ are independent identically distributed (i.i.d.) complex Gaussian random variables each with variance $\sigma_{v}^{2} . u$ represents the information-bearing signal, and $v$ represents the artificial noise. The received symbols at Bob and Eves become

$$
\begin{aligned}
& y_{B}=\boldsymbol{h} \boldsymbol{w}_{1} u+\boldsymbol{h} \boldsymbol{W}_{2} \boldsymbol{v}+n=\|\boldsymbol{h}\| u+n \\
& \boldsymbol{y}_{E}=\boldsymbol{G} \boldsymbol{w}_{1} u+\boldsymbol{G} \boldsymbol{W}_{2} \boldsymbol{v}+\boldsymbol{e}=\boldsymbol{g}_{1} u+\boldsymbol{G}_{2} \boldsymbol{v}+\boldsymbol{e}
\end{aligned}
$$

where we have defined $\boldsymbol{g}_{1}=\boldsymbol{G} \boldsymbol{w}_{1}$, and $\boldsymbol{G}_{2}=\boldsymbol{G} \boldsymbol{W}_{2}$.

We consider a total power per transmission denoted by $P$, that is, $P=\sigma_{u}^{2}+\left(N_{A}-1\right) \sigma_{v}^{2}$. Due to the normalization of the noise variance at Bob, we also refer to $P$ as the transmit SNR. One important design parameter is the ratio of power allocated to the information-bearing signal and the artificial noise. We denote the fraction of total power allocated to the information signal as $\phi$. Hence, we have the following relationships:

$$
\begin{aligned}
\sigma_{u}^{2} & =\phi P \\
\sigma_{v}^{2} & =(1-\phi) P /\left(N_{A}-1\right) .
\end{aligned}
$$

Since $\boldsymbol{h}$ is known by Alice, she can adaptively change the value of $\phi$ according to the instantaneous realization of $\boldsymbol{h}$. We refer to this strategy as the adaptive power allocation strategy. Alternatively, Alice can choose a fixed value for $\phi$, regardless of the instantaneous channel realization, which we refer to as the nonadaptive power allocation strategy. Note that Alice does not know $\boldsymbol{G}$ and, thus, equally distributes the transmit power among the artificial noise signal, as given by (6).

\section{SECRECy CAPACITy LOWER BOUND}

The secrecy capacity is the maximum transmission rate at which the intended receiver can decode the data with arbitrarily small error, whereas the mutual information between the transmitted message and the received signal at the eavesdropper is arbitrarily small. It is bounded by the difference in the capacity of the channel between Alice and Bob and that between Alice and Eve [2]. In this section, we derive a closed-form expression for an ergodic secrecy capacity lower bound with transmission of artificial noise.

The capacity of the channel between Alice and Bob is given by

$$
\begin{aligned}
C_{1} & =E_{\boldsymbol{h}}\left\{\log _{2}\left(1+\sigma_{u}^{2}\|\boldsymbol{h}\|^{2}\right)\right\} \\
& =E_{\boldsymbol{h}}\left\{\log _{2}\left(1+\phi P\|\boldsymbol{h}\|^{2}\right)\right\} .
\end{aligned}
$$

Without loss of generality, we normalize the variance of each element of $\boldsymbol{h}$ to unity. It is then easy to see that $\|\boldsymbol{h}\|^{2}$ follows a Gamma distribution with parameters $\left(N_{A}, 1\right)$. Therefore, for systems with nonadaptive power allocation strategy, we can rewrite (7) in integral form as

$$
\begin{aligned}
C_{1} & =\frac{1}{\ln 2} \int_{0}^{\infty} \ln (1+\phi P x) x^{N_{A}-1} \frac{\exp (-x)}{\Gamma\left(N_{A}\right)} d x \\
& =\frac{1}{\ln 2} \exp \left(\frac{z}{P}\right) \sum_{k=1}^{N_{A}} E_{k}\left(\frac{z}{P}\right)
\end{aligned}
$$

where $\Gamma(\cdot)$ is the Gamma function, $E_{n}(\cdot)$ is the generalized exponential integral, (8) is obtained using an integral identity given in [25], and we have defined $z=\phi^{-1}$.

Next, we study the capacity of the channel between Alice and the multiple colluding Eves. When multiple Eves are present, the noise at each Eve may be different. In addition, the receiver noise levels at Eves may not be known by Alice and Bob. To guarantee secure communication, it is therefore reasonable to consider the worst-case scenario where the noises at Eves are arbitrarily small. Note that this approach was also taken in [22]. In this case, we can normalize the distance of each Eve to make the variance of the elements of $G$ equal to unity without loss of generality. ${ }^{2}$

The noiseless eavesdropper assumption effectively gives an upper bound on the capacity of the channel between Alice and the multiple colluding Eves as

$$
\begin{aligned}
C_{2} & =E_{\boldsymbol{h}, \boldsymbol{g}_{1}, \boldsymbol{G}_{2}}\left\{\log _{2}\left|\boldsymbol{I}+\sigma_{u}^{2} \boldsymbol{g}_{1} \boldsymbol{g}_{1}^{\dagger}\left(\sigma_{v}^{2} \boldsymbol{G}_{2} \boldsymbol{G}_{2}^{\dagger}\right)^{-1}\right|\right\} \\
& =E_{\boldsymbol{h}, \boldsymbol{g}_{1}, \boldsymbol{G}_{2}}\left\{\log _{2}\left(1+\frac{N_{A}-1}{z-1} \boldsymbol{g}_{1}^{\dagger}\left(\boldsymbol{G}_{2} \boldsymbol{G}_{2}^{\dagger}\right)^{-1} \boldsymbol{g}_{1}\right)\right\}
\end{aligned}
$$

where we have again used $z=\phi^{-1}$. The expectation over $h$ in (9) is due to the fact that $z$ may be dependent on $\boldsymbol{h}$ (which happens when adaptive power allocation strategy is used). It is required in (9) that $\boldsymbol{G}_{2} \boldsymbol{G}_{2}^{\dagger}$ is invertible, which is guaranteed with the assumption of $N_{A}>N_{E}$. If the assumption is violated, then the colluding eavesdroppers are able to eliminate the artificial noise, resulting in $C_{2}=\infty$. Hence, we assume $N_{A}>N_{E}$ for guaranteeing secure communication.

Since $G$ has i.i.d. complex Gaussian entries, and $W$ is a unitary matrix, $\boldsymbol{G W}=\left[\boldsymbol{g}_{1} \boldsymbol{G}_{2}\right]$ also has i.i.d. complex Gaussian entries. Therefore, the elements of $\boldsymbol{g}_{1}$ and $\boldsymbol{G}_{2}$ are independent. As a result, the quantity $\boldsymbol{g}_{1}^{\dagger}\left(\boldsymbol{G}_{2} \boldsymbol{G}_{2}^{\dagger}\right)^{-1} \boldsymbol{g}_{1}$ is equivalent to the signal-to-interference ratio of a $N_{E}$-branch MMSE diversity combiner with $N_{A}-1$ interferers. The complementary cumulative distribution function of $X=\boldsymbol{g}_{1}^{\dagger}\left(\boldsymbol{G}_{2} \boldsymbol{G}_{2}^{\dagger}\right)^{-1} \boldsymbol{g}_{1}$ is given in [26] as

$$
R_{X}(x)=\frac{\sum_{k=0}^{N_{E}-1}\left(\begin{array}{c}
N_{A}-1 \\
k
\end{array}\right) x^{k}}{(1+x)^{N_{A}-1}} .
$$

\footnotetext{
${ }^{2}$ With the noiseless eavesdropper assumption, the capacity between Alice and each Eve is determined from the signal-to-artificial-noise ratio. Considering the signal reception at a particular Eve, both the information signal and the artificial noise are generated from the same source (Alice), and hence, their ratio is independent of the large-scale fading from Alice to Eve. That is to say, the signal-to-artificial-noise ratios are i.i.d. random variables for all Eves, regardless of their distances from Alice.
} 
Therefore, we can rewrite (9) in integral form as

$$
\begin{aligned}
C_{2}=E_{\boldsymbol{h}} & \left\{\int_{0}^{\infty} \log _{2}\left(1+\frac{N_{A}-1}{z-1} x\right) f_{X}(x) d x\right\} \\
=E_{\boldsymbol{h}} & \left\{\frac{1}{\ln 2} \int_{0}^{\infty} \frac{N_{A}-1}{z-1}\left(1+\frac{N_{A}-1}{z-1} x\right)^{-1} R_{X}(x) d x\right\} \\
=E_{\boldsymbol{h}} & \left\{\frac{1}{\ln 2} \sum_{k=0}^{N_{E}-1}\left(\begin{array}{c}
N_{A}-1 \\
k
\end{array}\right)\right. \\
& \left.\times \int_{0}^{\infty}\left(\frac{z-1}{N_{A}-1}+x\right)^{-1}(1+x)^{1-N_{A}} x^{k} d x\right\} \\
=E_{\boldsymbol{h}} & \left\{\frac{1}{\ln 2} \sum_{k=0}^{N_{E}-1}\left(\begin{array}{c}
N_{A}-1 \\
k
\end{array}\right) \frac{N_{A}-1}{z-1} B\left(k+1, N_{A}-1-k\right)\right. \\
& \left.\times{ }_{2} F_{1}\left(1, k+1 ; N_{A} ; \frac{z-N_{A}}{z-1}\right)\right\}
\end{aligned}
$$

where $f_{X}(x)$ denotes the probability density function of $X, B(\alpha, \beta)=\Gamma(\alpha) \Gamma(\beta) / \Gamma(\alpha+\beta)$ is the beta function, and ${ }_{2} F_{1}(\cdot)$ is the Gauss hypergeometric function. Note that (11) is obtained using integration by parts, and (12) is obtained using an integration identity given in [27].

After deriving expressions for $C_{1}$ and $C_{2}$, a lower bound on the ergodic secrecy capacity can now be obtained as $C=$ $\left[C_{1}-C_{2}\right]^{+}$, where $[\alpha]^{+}=\max \{0, \alpha\}$. This is a data rate that can always be guaranteed for secure communication (without knowing the noise level at Eves). For systems with adaptive power allocation, the ergodic secrecy capacity lower bound is given as

$$
\begin{aligned}
& C=\frac{1}{\ln 2}\left[E _ { \boldsymbol { h } } \left\{\ln \left(1+\frac{P}{z}\|\boldsymbol{h}\|^{2}\right)-\sum_{k=0}^{N_{E}-1}\left(\begin{array}{c}
N_{A}-1 \\
k
\end{array}\right) \frac{N_{A}-1}{z-1}\right.\right. \\
& \left.\left.\times B\left(k+1, N_{A}-1-k\right)_{2} F_{1}\left(1, k+1 ; N_{A} ; \frac{z-N_{A}}{z-1}\right)\right\}\right]^{+}
\end{aligned}
$$

where $z$ is a function of $\boldsymbol{h}$. For systems with nonadaptive power allocation, the ergodic secrecy capacity lower bound is given as

$$
\begin{aligned}
C= & \frac{1}{\ln 2}\left[\exp \left(\frac{z}{P}\right) \sum_{k=1}^{N_{A}} E_{k}\left(\frac{z}{P}\right)-\sum_{k=0}^{N_{E}-1}\left(\begin{array}{c}
N_{A}-1 \\
k
\end{array}\right) \frac{N_{A}-1}{z-1}\right. \\
& \left.\times B\left(k+1, N_{A}-1-k\right){ }_{2} F_{1}\left(1, k+1 ; N_{A} ; \frac{z-N_{A}}{z-1}\right)\right]^{+}
\end{aligned}
$$

where $z$ is a constant independent of $\boldsymbol{h}$.

Fig. 1 shows the ergodic secrecy capacity lower bound $C$ in (14) for systems with different numbers of antennas. We see that the presence of multiple colluding Eves dramatically re-

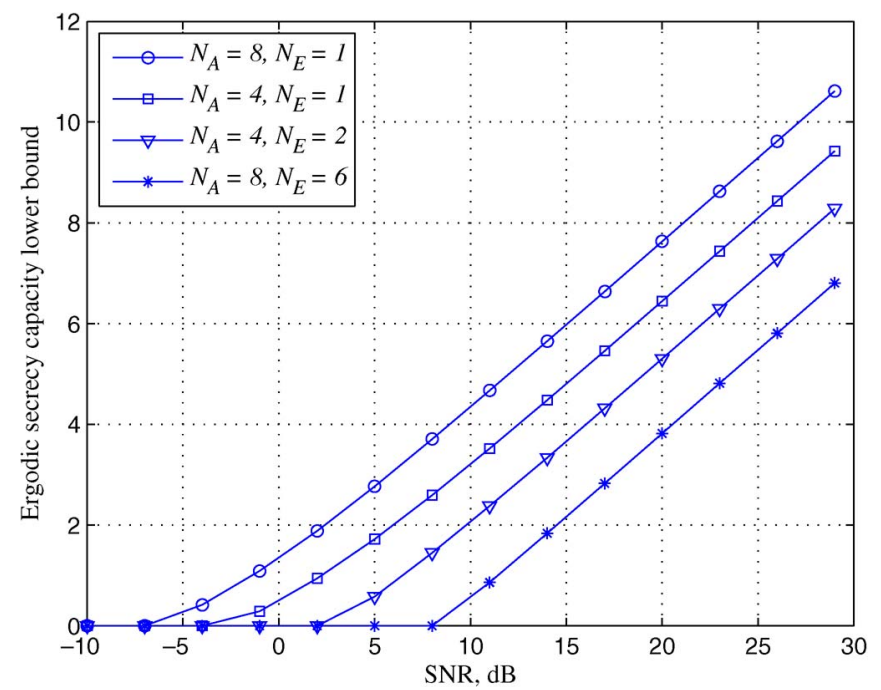

Fig. 1. Ergodic secrecy capacity lower bound $C$ in (14) versus SNR $P$ for systems with different numbers of antennas. The ratio of power allocation is set to $\phi=0.5$.

duces the secrecy rate compared with the case of noncolluding Eves. Furthermore, the secrecy rate quickly reduces to zero at low to moderate SNR.

In the following sections, we aim to give simplified or approximated expressions of the secrecy capacity lower bound in two special scenarios. These expressions will be used to obtain analytical results and useful insights on the optimal power allocation in Section IV. Note that the derived approximation may not be an achievable secrecy rate, although it is useful for the design of power allocation.

\section{A. Noncolluding Eavesdroppers}

In the case where Eves cannot collude, we have $N_{E}=1$. Then, $C_{2}$ in (12) reduces to

$$
\begin{aligned}
C_{2}=E_{\boldsymbol{h}} & \left\{\frac{1}{\ln 2} \frac{1}{z-1}{ }_{2} F_{1}\left(1,1 ; N_{A} ; \frac{z-N_{A}}{z-1}\right)\right\} \\
=E_{\boldsymbol{h}} & \left\{\frac{1}{\ln 2}\left(\frac{N_{A}-1}{N_{A}-z}\right)^{N_{A}-1}\right. \\
& \left.\times\left(\ln \left(\frac{N_{A}-1}{z-1}\right)-\sum_{l=1}^{N_{A}-2} \frac{1}{l}\left(\frac{N_{A}-z}{N_{A}-1}\right)\right)\right\}
\end{aligned}
$$

where (15) is obtained using an identity for the Gauss hypergeometric function derived in the Appendix. This can then be substituted into $C=\left[C_{1}-C_{2}\right]^{+}$to yield simplified expressions for the ergodic secrecy capacity lower bound.

\section{B. Large $N_{A}$ Analysis}

$C_{1}$ in (7) can be rewritten as

$$
\begin{aligned}
C_{1} & =E_{\boldsymbol{h}}\left\{\log _{2}\left(1+\frac{P}{z}\|\boldsymbol{h}\|^{2}\right)\right\} \\
& =\log _{2} N_{A}+E_{\boldsymbol{h}}\left\{\log _{2}\left(\frac{1}{N_{A}}+\frac{P}{z} \frac{\|\boldsymbol{h}\|^{2}}{N_{A}}\right)\right\} .
\end{aligned}
$$


The law of large numbers implies that $\lim _{N_{A} \rightarrow \infty}\|\boldsymbol{h}\|^{2} / N_{A}=$ 1 . Hence, we focus on the nonadaptive power allocation strategy where $z$ is a constant. In the large $N_{A}$ limit, we have

$$
\begin{aligned}
& \lim _{N_{A} \rightarrow \infty}\left(C_{1}-\log _{2} N_{A}\right) \\
& =\lim _{N_{A} \rightarrow \infty} E_{\boldsymbol{h}}\left\{\log _{2}\left(\frac{1}{N_{A}}+\frac{P}{z} \frac{\|\boldsymbol{h}\|^{2}}{N_{A}}\right)\right\} \\
& =\log _{2} \frac{P}{z} .
\end{aligned}
$$

That is to say, the difference between $C_{1}$ and $\log _{2} N_{A}$ approaches $\log _{2}(P / z)$ as $N_{A}$ increases. Therefore, in the large $N_{A}$ regime, we have ${ }^{3}$

$$
C_{1}=\log _{2}\left(\frac{N_{A} P}{z}\right)+o(1)
$$

From the law of large numbers, we also know that $\lim _{N_{A} \rightarrow \infty} \boldsymbol{G}_{2} \boldsymbol{G}_{2}^{\dagger} /\left(N_{A}-1\right)=\boldsymbol{I}$. Using (9) with the nonadaptive power allocation strategy, we have

$$
\begin{aligned}
& \lim _{N_{A} \rightarrow \infty} C_{2} \\
& =\lim _{N_{A} \rightarrow \infty} E_{\boldsymbol{g}_{1}, \boldsymbol{G}_{2}}\left\{\log _{2}\left(1+\frac{1}{z-1} \boldsymbol{g}_{1}^{\dagger}\left(\frac{\boldsymbol{G}_{2} \boldsymbol{G}_{2}^{\dagger}}{N_{A}-1}\right)^{-1} \boldsymbol{g}_{1}\right)\right\} \\
& =E_{\boldsymbol{g}_{1}}\left\{\log _{2}\left(1+\frac{1}{z-1}\left\|\boldsymbol{g}_{1}\right\|^{2}\right)\right\} \\
& =\frac{1}{\ln 2} \exp (z-1) \sum_{k=1}^{N_{E}} E_{k}(z-1)
\end{aligned}
$$

where $\left\|\boldsymbol{g}_{1}\right\|^{2}$ has a Gamma distribution with parameters $\left(N_{E}, 1\right)$. We can see from (19) that altering the number of antennas in Alice does not affect the channel capacity between Alice and Eves in the large $N_{A}$ limit.

The ergodic secrecy capacity lower bound in the large $N_{A}$ regime is then given by

$$
C=\frac{1}{\ln 2}\left[\ln \left(\frac{N_{A} P}{z}\right)-\exp (z-1) \sum_{k=1}^{N_{E}} E_{k}(z-1)+o(1)\right]^{+} .
$$

In Section IV, we will use the expression (dropping o(1)) in (20) as an approximation of the secrecy capacity lower bound for systems with large $N_{A}$ to study the optimal power allocation.

Fig. 2 shows the ergodic secrecy capacity lower bound $C$ in (14) as well as its large $N_{A}$ approximation in (20). We see that (14) converges to (20) as $N_{A}$ increases. The convergence is fast for a small number of colluding Eves, e.g., $N_{E}=2$, and is slow for a large number of colluding Eves, e.g., $N_{E}=6$.

\footnotetext{
${ }^{3}$ The notation $f(x)=o(g(x))$ implies that $\lim _{x \rightarrow \infty}(f(x) / g(x))=0$. This limit is taken w.r.t. $N_{A}$ in (18).
}

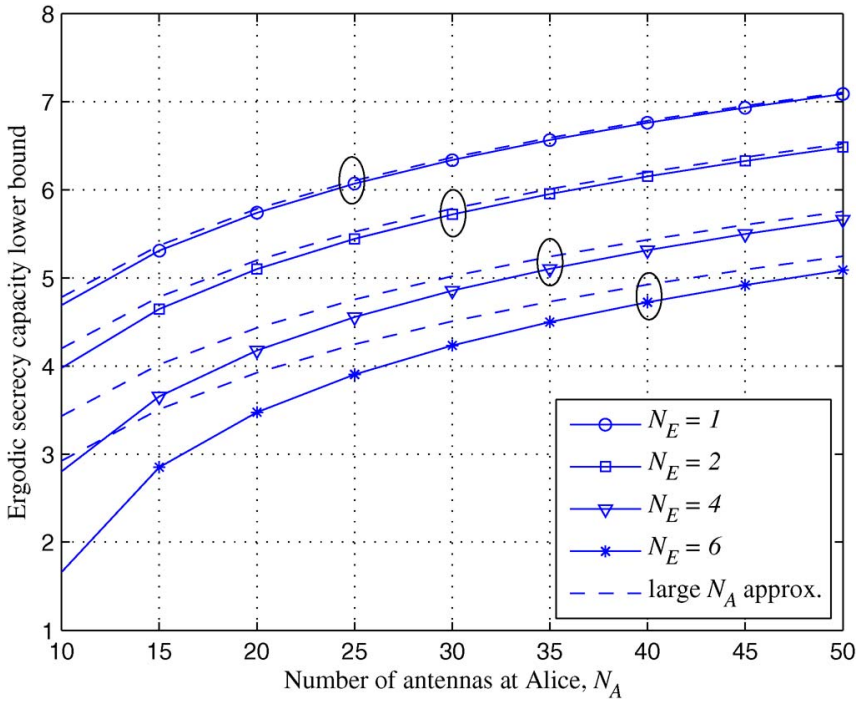

Fig. 2. Ergodic secrecy capacity lower bound $C$ in (14) at $10 \mathrm{~dB}$ versus the number of antennas at Alice $N_{A}$ for systems with different numbers of colluding eavesdroppers. The large $N_{A}$ approximations of $C$ in (20) are shown as dashed lines. The ratio of power allocation is set to $\phi=0.5$.

\section{Optimal Power Allocation}

In this section, we study the optimal power allocation between the information-bearing signal and the artificial noise. As we have discussed, the power allocation strategy can be either adaptive or nonadaptive. The former depends on every realization of the channel gain, whereas the latter is fixed for all channel realizations. The objective function for this optimization problem is the ergodic secrecy capacity lower bound. The closed-form expressions derived in the previous section greatly reduce the computational complexity of the optimization process. In the following, we first study the case of noncolluding eavesdroppers and then look at the case of colluding eavesdroppers.

\section{A. Noncolluding Eavesdropper Case}

The optimal value of $\phi$ or $z$ can easily be found numerically using the capacity lower bound expressions derived in Section III. Moreover, these expressions enable us to analytically obtain useful insights into the optimal $z$ in the high SNR regime as follows.

In the high $\mathrm{SNR}$ regime, i.e., $P \gg 1, C_{1}$ in (7) can be approximated as

$$
\begin{aligned}
C_{1} & \approx E_{\boldsymbol{h}}\left\{\log _{2}\left(\frac{P}{z}\|\boldsymbol{h}\|^{2}\right)\right\} \\
& =E_{\boldsymbol{h}}\left\{\log _{2}\left(P\|\boldsymbol{h}\|^{2}\right)\right\}-E_{\boldsymbol{h}}\left\{\log _{2} z\right\} .
\end{aligned}
$$

We see in (21) that $E_{\boldsymbol{h}}\left\{\log _{2}\left(P\|\boldsymbol{h}\|^{2}\right)\right\}$ is a constant, and $E_{\boldsymbol{h}}\left\{\log _{2} z\right\}$ does not directly depend on $\boldsymbol{h}$, although $z$ may be a function of $\boldsymbol{h}$. Therefore, the high SNR approximation of the secrecy capacity lower bound does not have $h$ in its expression (except for the expectation over $\boldsymbol{h}$ ). Consequently, for any value of $\boldsymbol{h}$, the optimal $z$ that maximizes the high SNR approximation of the secrecy capacity lower bound is the same. In other words, the value of $\boldsymbol{h}$ is irrelevant in finding 


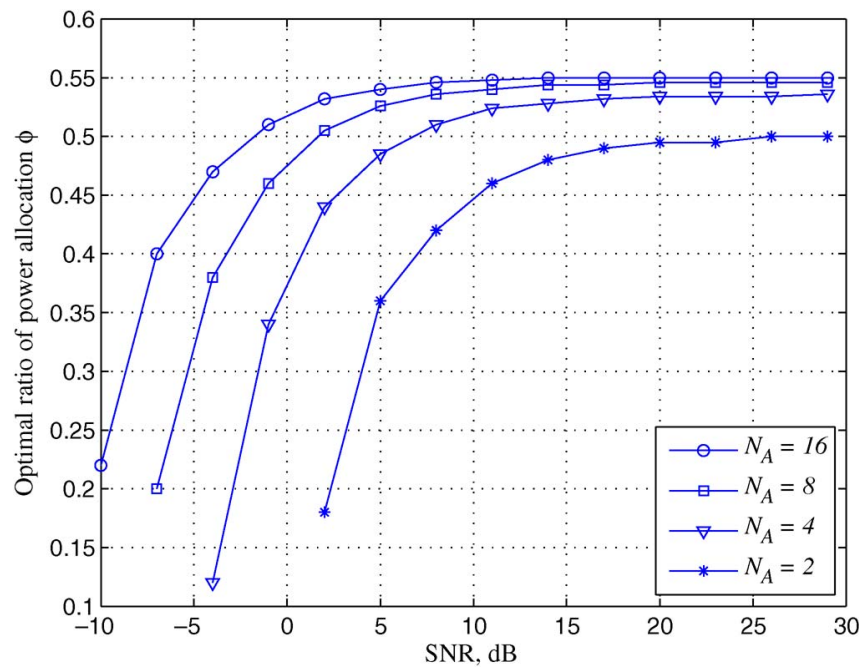

Fig. 3. Optimal ratio of power allocation $\phi$ versus SNR $P$ for different numbers of antennas at Alice $N_{A}$. The nonadaptive power allocation strategy is used. The values of $\phi$ are shown for SNRs at which the ergodic secrecy capacity lower bound is positive.

the optimal power allocation. Therefore, the adaptive power allocation strategy does not need to be considered at high SNR.

The optimal value of $z$ in the high SNR regime satisfies

$$
\frac{d C}{d z}=\frac{d C_{1}}{d z}-\frac{d C_{2}}{d z}=-\frac{1}{z \ln 2}-\frac{d C_{2}}{d z}=0
$$

where the derivative of $C_{2}$ w.r.t. $z$ can be computed in closedform using (15).

In the special case of $N_{A}=2,(22)$ reduces to

$$
-\frac{1}{z}-\frac{1}{(z-2)(z-1)}+\frac{\ln (z-1)}{(z-2)^{2}}=0 .
$$

The solution to the preceding equation is given by $z=2$. It can be shown that $\lim _{z \rightarrow 2}\left(d^{2} C / d z^{2}\right)<0$. Hence, the optimal ratio of power allocation is given by $\phi=0.5$, that is to say, equal power allocation between the information signal and the artificial noise is the optimal strategy in the high SNR regime for $N_{A}=2$.

For large $N_{A}$, using (19) with $N_{E}=1$, we have

$$
\begin{aligned}
\frac{d C_{2}}{d z} & =\frac{1}{\ln 2}\left(\exp (z-1) E_{1}(z-1)-\exp (z-1) E_{0}(z-1)\right) \\
& =\frac{1}{\ln 2}\left(\exp (z-1) E_{1}(z-1)-(z-1)^{-1}\right)
\end{aligned}
$$

Hence, the optimal value of $z$ satisfies

$$
-\frac{1}{z}-e^{z-1} E_{1}(z-1)+\frac{1}{z-1}=0
$$

which gives $z=1.80$. It can be shown that at $z=1.80$, $\left(d^{2} C / d z^{2}\right)<0$. Hence, the optimal ratio of power allocation is given by $\phi=0.55$ in the high SNR regime for sufficiently large $N_{A}$. We see that the difference between the optimal values of $\phi$ for the smallest $N_{A}$ (i.e., $N_{A}=2$ ) and asymptotically large $N_{A}$ is very small.

Fig. 3 shows the optimal values of $\phi$ using the nonadaptive power allocation strategy for systems with different numbers

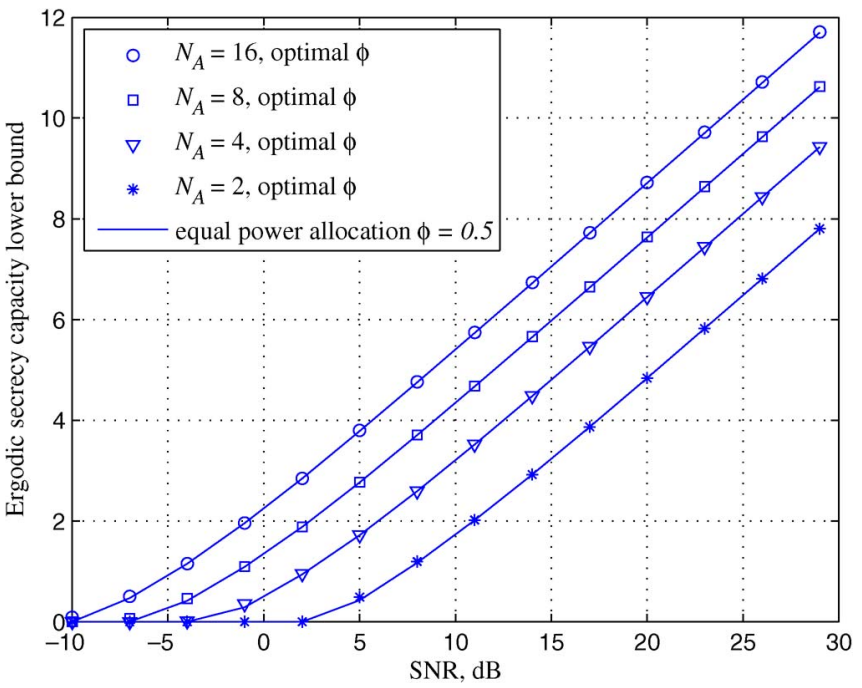

Fig. 4. Ergodic secrecy capacity lower bound $C$ in (14) versus SNR $P$ for different numbers of antennas at Alice $N_{A}$. The nonadaptive power allocation strategy is used. The ergodic secrecy capacity lower bound with equal power allocation for each case, which is indicated by solid line, is also shown for comparison.

of antennas at Alice $N_{A}$. The values of $\phi$ are shown for SNRs at which the ergodic secrecy capacity lower bound is positive. The general trend is that more power needs to be allocated to the information signal as the SNR or $N_{A}$ increases. In the high SNR regime, we see that the optimal values of $\phi$ converge to constant values. For $N_{A}=2$, the optimal value of $\phi$ converges to 0.5 , which agrees with our analytical result. Furthermore, this constant value only increases slightly with $N_{A}$, and the maximum value is 0.55 , which agrees with our large $N_{A}$ analysis. These observations suggest that a near-optimal yet simple power allocation strategy at moderate to high SNR values is the equal power allocation between the information signal and the artificial noise.

Fig. 4 shows the ergodic secrecy capacity lower bound $C$ in (14) with the optimized $\phi$ using the nonadaptive power allocation strategy. For comparison, we also include the capacity lower bound with equal power allocation, i.e., $\phi=0.5$, which is indicated by solid lines. We see that the equal power allocation strategy achieves nearly the same secrecy rate as the optimal nonadaptive power allocation in all cases over a wide range of SNR values. This confirms that equal power allocation is a simple and generic strategy, which yields close to optimal performance in terms of the derived achievable secrecy rate.

Fig. 5 shows the ergodic secrecy capacity lower bound $C$ in (13) and (14) with the optimized $\phi$ using both adaptive and nonadaptive power allocation strategies. For adaptive power allocation, we apply a linear search on $\phi$ to find the optimal value that maximizes the secrecy capacity lower bound for each realization of $\boldsymbol{h}$. The maximum value of the secrecy capacity lower bound for each channel realization is recorded, and the ergodic secrecy capacity lower bound is then computed using the distribution of $\boldsymbol{h}$. We see that there is no difference between the secrecy rate achieved by the adaptive and nonadaptive strategies over a wide range of SNR values. The adaptive strategy only gives marginal advantage when the secrecy rate is close to zero. This result suggests that the nonadaptive power allocation 


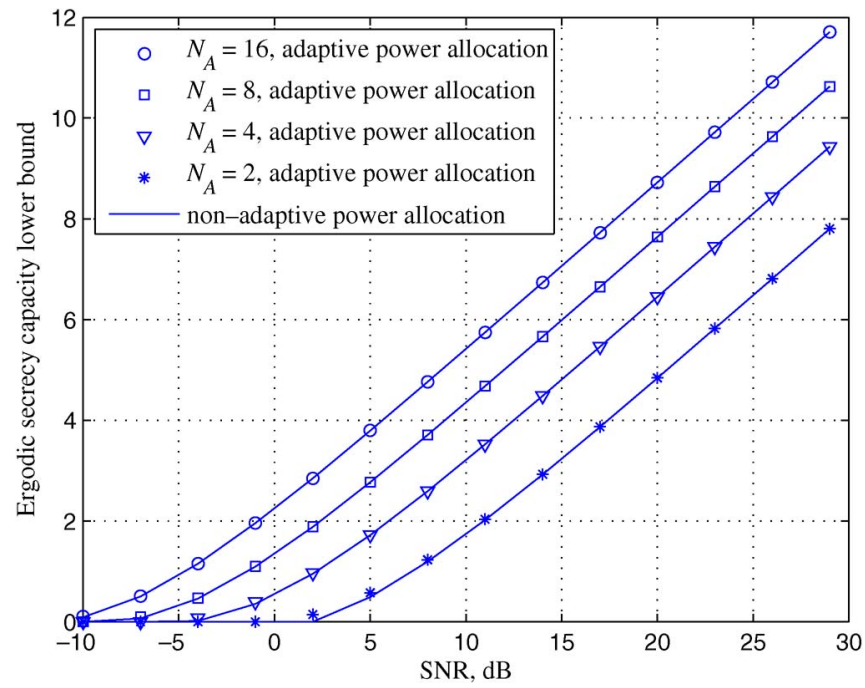

Fig. 5. Ergodic secrecy capacity lower bound $C$ in (13) and (14) versus SNR $P$ for different numbers of antennas at Alice $N_{A}$. Both the adaptive and nonadaptive power allocation strategies are used, which are indicated by markers and lines, respectively.

strategy is sufficient to achieve almost the best possible secrecy rate performance. ${ }^{4}$ For this reason, we will only focus on the nonadaptive scheme in the rest of this paper.

\section{B. Colluding Eavesdropper Case}

As we have seen in Fig. 1, the presence of multiple colluding Eves severely degrades the secrecy rate. Therefore, it is essential for Alice to have a relatively large number of antennas to maintain a good secure communication link. For any value of $N_{E}$, the optimal value of $\phi$ or $z$ can easily be found numerically using the closed-form capacity lower bound expression given in Section III. As the number of antennas at Alice is desired to be large, we carry out large $N_{A}$ analysis to obtain an asymptotic result on optimal $z$ in the high SNR regime as follows.

In the high SNR regime with large $N_{A}, C$ in (20) can be approximated as

$$
C \approx \frac{1}{\ln 2}\left[\ln \left(N_{A} P\right)-\ln z-\exp (z-1) \sum_{k=1}^{N_{E}} E_{k}(z-1)\right] .
$$

By taking the derivative of $C$ w.r.t. $z$, the optimal $z$ satisfies

$$
-\frac{1}{z}-e^{z-1} E_{N_{E}}(z-1)+\frac{1}{z-1}=0 .
$$

Using $e^{z-1} E_{N_{E}}(z-1) \approx\left(z-1+N_{E}\right)^{-1}$ from [28], which is accurate when either $N_{E}$ or $z$ is large, (27) reduces to

$$
-\frac{1}{z}-\frac{1}{z-1+N_{E}}+\frac{1}{z-1}=0 .
$$

Hence, the optimal $z$ is given by

$$
z^{*}=1+\sqrt{N_{E}} .
$$

\footnotetext{
${ }^{4}$ The same result is found for the colluding Eves case. The numerical results are omitted for brevity.
}

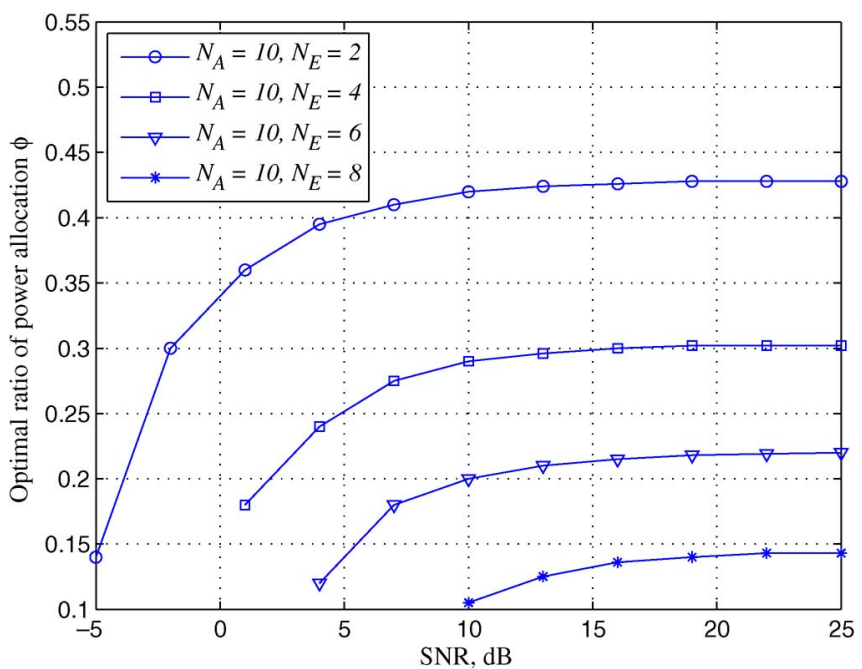

Fig. 6. Optimal ratio of power allocation $\phi$ versus SNR $P$ for systems with different numbers of colluding Eves $N_{E}$. The values of $\phi$ are shown for SNRs at which the ergodic secrecy capacity lower bound is positive.

From (29), we see that the optimal value of $z$ only depends on $N_{E}$ in the high SNR and large antenna regime. Moreover, (29) suggests that more power should be used to generate artificial noise when the number of Eves increases.

Fig. 6 shows the optimal value of $\phi$ for systems with different numbers of colluding Eves $N_{E}$. Similar to the noncolluding Eves case, we see that more power should be used to transmit the information signal as the SNR increases. The optimal value of $\phi$ stays constant in the high SNR regime. Furthermore, the optimal value of $\phi$ for the colluding Eves case is usually much smaller than 0.5, i.e., equal power allocation, which is near optimal for noncolluding Eves case. In particular, the optimal $\phi$ reduces as $N_{E}$ grows, which implies that more power should be allocated to generate the artificial noise as the number of colluding Eves increases. This observation agrees with our analytical insight and intuition.

Fig. 7 shows the ergodic secrecy capacity lower bound $C$ in (14) for systems with different $N_{E}$. Here, we investigate sensitivity in the secrecy rate to the design of power allocation. Consider a scenario where the total number of Eves that can collude is 8 , and hence, Alice has optimized $\phi$ for $N_{E}=8$. When $N_{E}$ changes, the power allocation parameter $\phi$ does not need to be optimized again as long as $N_{E}$ stays reasonably close to 8 , e.g., $N_{E}=6$, since the value of $\phi$ optimized for $N_{E}=8$ still works well for $N_{E}=6$ (with a power loss of $0.2 \mathrm{~dB}$ ), as shown in Fig. 7. However, redesigning of $\phi$ becomes important when $N_{E}$ is considerably different from 8 , e.g., $N_{E}=2$ to 4 . For example, if $N_{E}$ changes from 8 to 4 , then a power loss of approximately $1 \mathrm{~dB}$ will incur if Alice still uses the value of $\phi$ optimized for $N_{E}=8$, as shown in Fig. 7.

We also provide numerical verification of the optimal power allocation obtained from the large antenna approximation in the high SNR regime. Fig. 8 shows the ratio of power allocation $\phi$ at $20 \mathrm{~dB}$ versus the number of antennas at Alice $N_{A}$ for systems with different numbers of colluding Eves $N_{E}$. For a fixed $N_{E}$, we see that the optimal value of $\phi$ increases with $N_{A}$ and reaches a constant value when $N_{A}$ is sufficiently large. This agrees with our analytical insight that the optimal power 


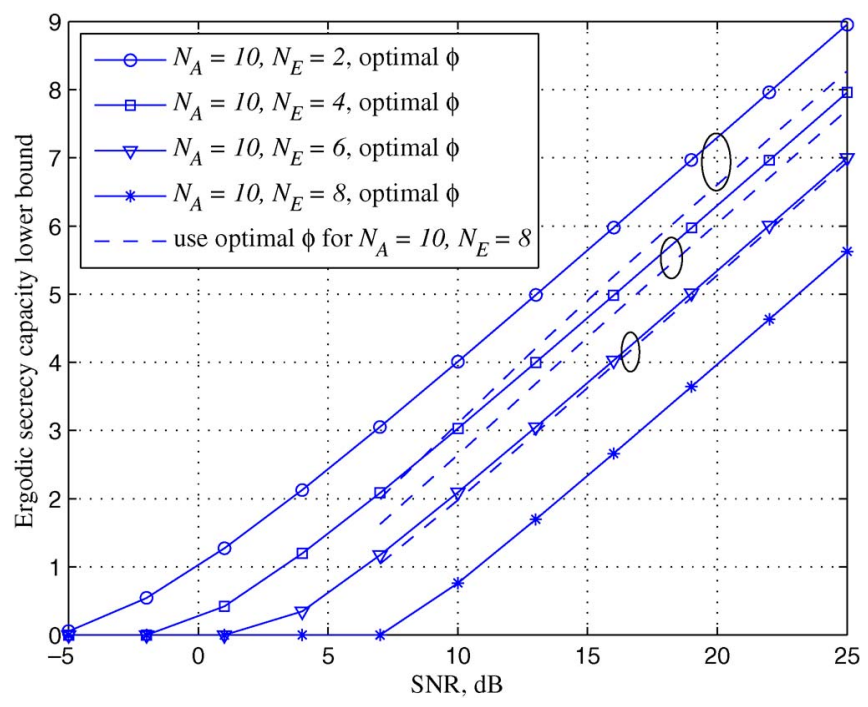

Fig. 7. Ergodic secrecy capacity lower bound $C$ in (14) versus SNR $P$ for systems with different numbers of colluding Eves $N_{E}$. The solid lines with markers indicate $C$ achieved with optimal values of $\phi$ for the corresponding system. The dashed lines indicate $C$ achieved with value of $\phi$ optimized for $N_{E}=8$, which represents the case where the power allocation was initially designed for $N_{E}=8$, but the current value of $N_{E}$ reduces from 8 , and the power allocation is not redesigned.

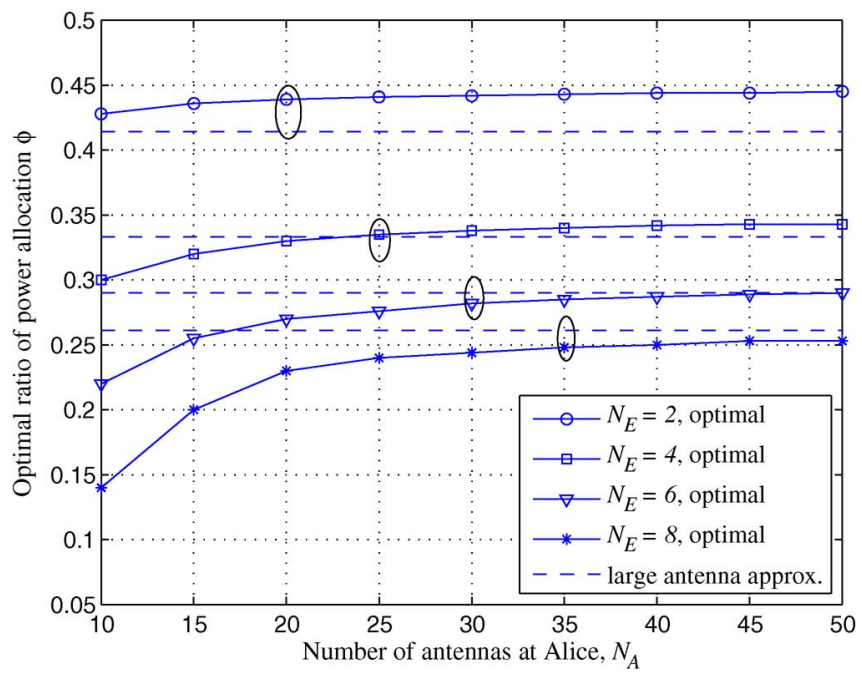

Fig. 8. Ratio of power allocation $\phi$ at $20 \mathrm{~dB}$ versus the number of antennas at Alice $N_{A}$ for systems with different numbers of colluding Eves $N_{E}$. The solid lines with markers indicate optimal values of $\phi$, whereas the dashed lines indicate the values of $\phi$ from the large antenna approximation given in (29).

allocation depends on $N_{E}$ but not on $N_{A}$ when $N_{A}$ is large. The asymptotic constant value of $\phi$ is close to the analytical value given in (29) obtained from the large antenna approximation.

In the system model, we have assumed fixed power transmission over time. When variable power transmission is allowed subject to an average power constraint, the achievable secrecy rate can be increased by having temporal power allocation according to the channel gain at each time instant. From the derived secrecy rate expression, we see that the transmit power only affects the transmission rate between Alice and Bob. The existing study on the point-to-point channel capacity, e.g., in [29], showed that the temporal power optimization gives little capacity gain, provided that the spatial power optimization is used.

In reality, noise is always present at the eavesdroppers, and hence, the designed power allocation strategy is not the optimal strategy in practice. If the eavesdroppers' noise levels are known to the transmitter and, hence, are taken into account in the secrecy rate expression, the efficiency of using artificial noise in degrading the capacity between Alice and Eve is reduced. Therefore, more power should be used to transmit the information signal.

\section{CRitical Signal-to-Noise Ratio FOR SECURE COMMUNICATIONS}

Another important aspect of secure communication is the minimum SNR required for a positive secrecy rate, which is a critical parameter in wideband communications. With the closed-form expression of the secrecy capacity lower bound derived in Section III, one can numerically find the critical SNR with low computational complexity. In this section, we derive a closed-form upper bound on the critical SNR, which is useful in the design of wideband communications.

Using properties of the exponential integral function in [28], (8) can be bounded as

$$
C_{1}>\frac{1}{\ln 2} \sum_{k=1}^{N_{A}} \frac{1}{\frac{z}{P}+k}
$$

which is asymptotically tight as the SNR approaches zero, i.e., $P \rightarrow 0$. Using the convexity of (30) in $k$, we can further bound $C_{1}$ as

$$
C_{1}>\frac{1}{\ln 2} \frac{N_{A}}{\frac{z}{P}+\frac{N_{A}+1}{2}}
$$

which is also asymptotically tight as the SNR approaches zero. Using the lower bound on $C_{1}$ in (31) and $C_{2}$ in (12), the ergodic secrecy capacity lower bound can further be bounded as

$$
\begin{aligned}
C> & \frac{1}{\ln 2} \frac{N_{A}}{\frac{z}{P}+\frac{N_{A}+1}{2}}-\frac{1}{\ln 2} \sum_{k=0}^{N_{E}-1}\left(\begin{array}{c}
N_{A}-1 \\
k
\end{array}\right) \frac{N_{A}-1}{z-1} \\
& \times B\left(k+1, N_{A}-1-k\right)_{2} F_{1}\left(1, k+1 ; N_{A} ; \frac{z-N_{A}}{z-1}\right) .
\end{aligned}
$$

The critical SNR, which is denoted by $P_{C}$, is the SNR at which $C$ drops to zero. With the lower bound on $C$ given in (32), an upper bound on $P_{C}$ can be found in (33), shown at the bottom of the next page. In the case of noncolluding eavesdroppers, i.e., $N_{E}=1$, (33) reduces to (34), also shown at the bottom of the next page. The upper bound in (33) or (34) indicates a minimum SNR that guarantees a positive secrecy rate. Since (33) and (34) are asymptotically tight at low SNR, they can be used to fine tune the power allocation parameter $z$ to minimize $P_{C}$.

Fig. 9 shows the critical SNR $P_{C}$ versus number of antennas at Alice $N_{A}$ for systems with different numbers of colluding Eves $N_{E}$. The power allocation is set to $\phi=0.2$ in all cases. The general trend is that $P_{C}$ decreases as $N_{A}$ increases, and a 


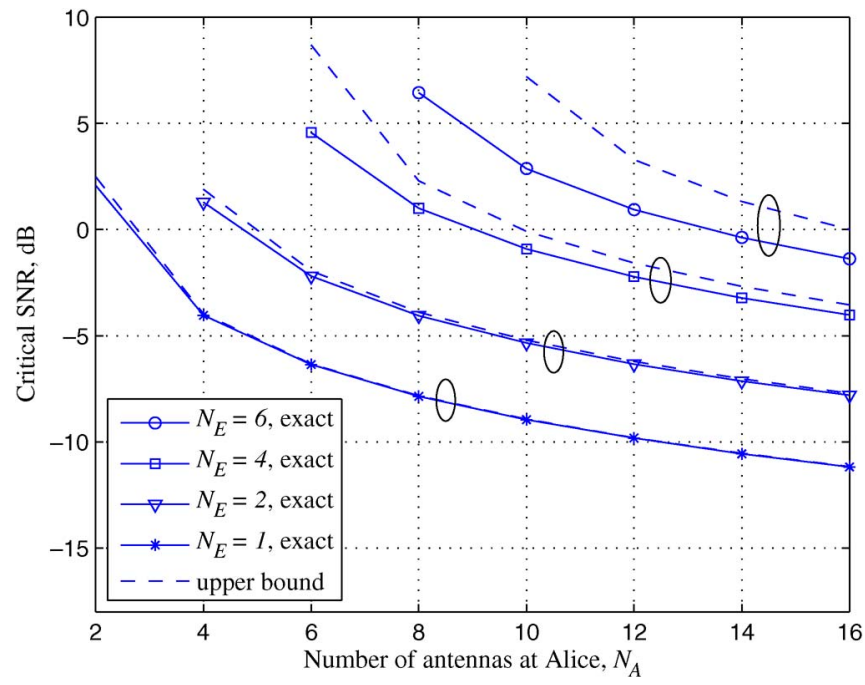

Fig. 9. Critical SNR $P_{C}$ versus number of antennas at Alice $N_{A}$ for systems with different numbers of colluding Eves $N_{E}$. The ratio of power allocation is set to $\phi=0.2$. The solid lines with markers indicate the exact value of $P_{C}$, whereas the dashed lines indicate the analytical upper bound given in (33).

higher $P_{C}$ is required when $N_{E}$ increases. These observations agree with intuition. Furthermore, we see that the analytical upper bound on $P_{C}$ is very accurate for the case of noncolluding Eves. For the case of colluding Eves, the upper bound is reasonably accurate when $P_{C}<0 \mathrm{~dB}$. The difference between the exact value of $P_{C}$ and its upper bound gradually increases as $N_{E}$ increases, which is mainly due to the increase in $P_{C}$. When $N_{E}$ is relatively large, e.g., $N_{E}=6$, one should allocate more power to generate the artificial noise (i.e., reduce $\phi$ ), as suggested in Fig. 6, to achieve a lower $P_{C}$, which in turn makes the bound tighter.

\section{EFFECT OF IMPERFECT CHANNEl STATE INFORMATION}

So far, we have assumed that the CSI can perfectly be obtained at Alice and Bob. In this section, we investigate the effect of imperfect CSI by considering channel estimation errors. With imperfect CSI, the beamforming transmission from Alice to Bob is designed based on the estimated channel gains rather than the true channel gains. Therefore, the artificial noise leaks into Bob's channel.
To incorporate imperfect CSI, we consider that Bob performs MMSE channel estimation. Therefore, we have

$$
\begin{gathered}
\boldsymbol{h}=\hat{\boldsymbol{h}}+\tilde{\boldsymbol{h}} \\
\sigma_{h}^{2}=\sigma_{\hat{h}}^{2}+\sigma_{\tilde{h}}^{2}
\end{gathered}
$$

where $\hat{\boldsymbol{h}}$ denotes the channel estimate, and $\tilde{\boldsymbol{h}}$ denotes the estimation error. $\sigma_{h}^{2}$ denotes the variance of each element in $\boldsymbol{h}$. $\sigma_{\hat{h}}^{2}$ and $\sigma_{\tilde{h}}^{2}$ denote the variance of each element in $\hat{\boldsymbol{h}}$ and $\tilde{\boldsymbol{h}}$, respectively. As a general property of the MMSE estimator for Gaussian signals [30], $\hat{\boldsymbol{h}}$ and $\tilde{\boldsymbol{h}}$ are uncorrelated, with each having i.i.d. complex Gaussian entries.

Similar to our system model in Section II, we assume that knowledge of $\hat{\boldsymbol{h}}$ is available at Alice and Eves. Therefore, the beamforming vector becomes $\boldsymbol{w}_{1}=\hat{\boldsymbol{h}}^{\dagger} /\|\hat{\boldsymbol{h}}\|$, and the received symbol at Bob is given by

$$
y_{B}=\hat{\boldsymbol{h}} \boldsymbol{x}+\tilde{\boldsymbol{h}} \boldsymbol{x}+n=\|\hat{\boldsymbol{h}}\| u+\tilde{\boldsymbol{h}} \boldsymbol{W}\left[u \boldsymbol{v}^{T}\right]^{T}+n .
$$

A capacity lower bound for the channel between Alice and Bob can be obtained by considering $\tilde{\boldsymbol{h}} \boldsymbol{W}\left[u \boldsymbol{v}^{T}\right]^{T}+n$ as the worst-case Gaussian noise [31]. Note that $\boldsymbol{W}$ is a unitary matrix; hence, $\tilde{\boldsymbol{h}} \boldsymbol{W}$ has the same distribution as $\tilde{\boldsymbol{h}}$ [32]. Therefore, the ergodic capacity lower bound for the channel between Alice and Bob is given by

$$
\hat{C}_{1}=E_{\hat{\boldsymbol{h}}}\left\{\log _{2}\left(1+\frac{\sigma_{u}^{2}\|\hat{\boldsymbol{h}}\|^{2}}{\sigma_{\tilde{h}}^{2} P+1}\right)\right\} .
$$

With $\sigma_{h}^{2}$ normalized to unity, we have $\sigma_{\hat{h}}^{2}=1-\sigma_{\tilde{h}}^{2}$. Since the elements of $\hat{\boldsymbol{h}}$ are i.i.d. complex Gaussian, $\|\hat{\boldsymbol{h}}\|^{2}$ is a sum of i.i.d. exponential distributed random variables, which follows a Gamma distribution with parameter $\left(N_{A}, 1-\sigma_{\tilde{h}}^{2}\right)$. Therefore, we obtain a closed-form expression for $\hat{C}_{1}$ as

$$
\hat{C}_{1}=\frac{1}{\ln 2} \exp \left(z \frac{\sigma_{\tilde{h}}^{2}+P^{-1}}{1-\sigma_{\tilde{h}}^{2}}\right) \sum_{k=1}^{N_{A}} E_{k}\left(z \frac{\sigma_{\tilde{h}}^{2}+P^{-1}}{1-\sigma_{\tilde{h}}^{2}}\right)
$$

The presence of channel estimation errors does not affect the signal reception at Eve given in (4). Therefore, the ergodic

$$
P_{C}<z\left[\frac{N_{A}}{\sum_{k=0}^{N_{E}-1}\left(\begin{array}{c}
N_{A}-1 \\
k
\end{array}\right) \frac{N_{A}-1}{z-1} B\left(k+1, N_{A}-1-k\right)_{2} F_{1}\left(1, k+1 ; N_{A} ; \frac{z-N_{A}}{z-1}\right)}-\frac{N_{A}+1}{2}\right]^{-1}
$$

$$
P_{C}<z\left[\frac{N_{A}}{\left(\frac{N_{A}-1}{N_{A}-z}\right)^{N_{A}-1}\left(\ln \left(\frac{N_{A}-1}{z-1}\right)-\sum_{l=1}^{N_{A}-2} \frac{1}{l}\left(\frac{N_{A}-z}{N_{A}-1}\right)^{l}\right)}-\frac{N_{A}+1}{2}\right]^{-1}
$$


secrecy capacity lower bound can be obtained by subtracting $C_{2}$ from $\hat{C}_{1}$ as

$$
\begin{aligned}
C=\frac{1}{\ln 2}[ & \exp \left(z \frac{\sigma_{\tilde{h}}^{2}+P^{-1}}{1-\sigma_{\tilde{h}}^{2}}\right) \sum_{k=1}^{N_{A}} E_{k}\left(z \frac{\sigma_{\tilde{h}}^{2}+P^{-1}}{1-\sigma_{\tilde{h}}^{2}}\right) \\
& -\sum_{k=0}^{N_{E}-1}\left(\begin{array}{c}
N_{A}-1 \\
k
\end{array}\right) \frac{N_{A}-1}{z-1} B\left(k+1, N_{A}-1-k\right) \\
& \left.\times{ }_{2} F_{1}\left(1, k+1 ; N_{A} ; \frac{z-N_{A}}{z-1}\right)\right]^{+} .
\end{aligned}
$$

Following the steps in Section $\mathrm{V}$, we can also bound $C$ to obtain an upper bound on the critical SNR for secure communication with channel estimation errors as

$$
\begin{aligned}
C> & \frac{1}{\ln 2} \frac{N_{A}}{z \frac{\sigma_{\tilde{h}}^{2}+P^{-1}}{1-\sigma_{\tilde{h}}^{2}}+\frac{N_{A}+1}{2}}-\frac{1}{\ln 2} \sum_{k=0}^{N_{E}-1}\left(\begin{array}{c}
N_{A}-1 \\
k
\end{array}\right) \frac{N_{A}-1}{z-1} \\
& \times B\left(k+1, N_{A}-1-k\right){ }_{2} F_{1}\left(1, k+1 ; N_{A} ; \frac{z-N_{A}}{z-1}\right) .
\end{aligned}
$$

The upper bound on the critical SNR is then given in (42), shown at the bottom of the page, which is asymptotically tight at low SNR.

We now present numerical results on the optimal power allocation as well as the critical SNR in the presence of channel estimation errors. For brevity, we focus on the case of noncolluding eavesdroppers. The trends on the effect of channel estimation errors observed in the following results also apply to the case of colluding eavesdroppers.

Fig. 10 shows the optimal ratio of power allocation $\phi$ with different channel estimation error variances $\sigma_{\tilde{h}}^{2}$. We see that the channel estimation error has noticeable impact on the value of $\phi$, particularly for a small number of antennas at Alice, e.g., $N_{A}=2$. The general trend is that less power should be allocated to information signal as the channel estimation error increases. This is mainly due to the fact that the efficiency of improving Bob's signal reception by boosting the transmit power of the information signal reduces as the channel estimation error increases. On the other hand, the efficiency of degrading Eve's signal reception by boosting the transmit power of the artificial noise stays the same, regardless of the channel estimation error. Hence, it is better to create more noise for Eves than to increase the signal strength for Bob if the CSI is not accurately obtained.

In practical systems, the channel estimation error usually reduces as the SNR increases, although their exact relationship depends on the training design. From Fig. 10, we can expect

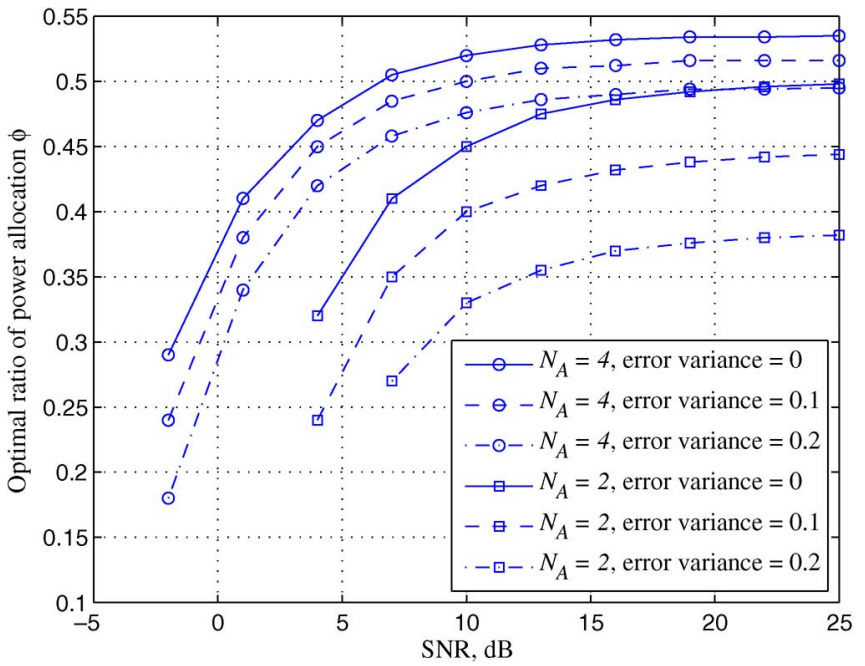

Fig. 10. Optimal ratio of power allocation $\phi$ versus SNR $P$ for different numbers of antennas at Alice $N_{A}$ and different variances of the channel estimation errors $\sigma_{\tilde{h}}^{2}$. The values of $\phi$ are shown for SNRs at which the ergodic

\begin{tabular}{|c|c|c|c|c|c|c|}
\hline & \multirow{2}{*}{\begin{tabular}{|c|} 
Error variance \\
$\sigma_{\tilde{h}}^{2}$ \\
\end{tabular}} & \multicolumn{5}{|c|}{ Number of antennas $N_{A}$} \\
\hline & & 2 & 4 & 6 & 8 & 10 \\
\hline Exact & 0 & 3.01 & -2.62 & -4.89 & -6.36 & -7.45 \\
\hline Upper bound & 0 & 6.02 & -1.97 & -4.46 & -6.01 & -7.14 \\
\hline Exact & 0.1 & 4.56 & -1.88 & -4.27 & -5.79 & -6.90 \\
\hline Upper bound & 0.1 & 9.03 & -1.20 & -3.83 & -5.43 & -6.59 \\
\hline Exact & 0.2 & 6.99 & -1.01 & -3.55 & -5.13 & -6.28 \\
\hline Upper bound & 0.2 & $\infty$ & -0.26 & -3.08 & -4.76 & -5.96 \\
\hline
\end{tabular}
secrecy capacity lower bound is positive.

TABLE I

CRitical SNR (In Decibels) For SeCuRe Communications With EQUal POWER Allocation

that at low to moderate SNR, where the channel estimation error is usually noticeable, the optimal power allocation is very different from that in the perfect CSI case. While at high SNR, where the channel estimation error is usually small, the optimal power allocation is expected to be very close to that of the perfect CSI case. Therefore, in practical systems, it is important to take channel estimation error into account when designing the power allocation at relatively low SNR.

Table I lists the exact values of the critical SNR $P_{C}$, as well as the closed-form upper bound given in (42) with $\phi=0.5$. The general trend is that the critical SNR increases as the channel estimation error increases, which agrees with intuition. The upper bound gets tighter as $P_{C}$ reduces (or $N_{A}$ increases) and is accurate for $N_{A} \geq 4$, with an error of less than $1 \mathrm{~dB}$.

\section{CONCLUSION}

In this paper, we have considered secure communication in the wireless fading environment in the presence of

$$
P_{C}<\left[\frac{1-\sigma_{\tilde{h}}^{2}}{z}\left(\frac{N_{A}}{\sum_{k=0}^{N_{E}-1}\left(\begin{array}{c}
N_{A}-1 \\
k
\end{array}\right) \frac{N_{A}-1}{z-1} B\left(k+1, N_{A}-1-k\right)_{2} F_{1}\left(1, k+1 ; N_{A} ; \frac{z-N_{A}}{z-1}\right)}-\frac{N_{A}+1}{2}\right)-\sigma_{\tilde{h}}^{2}\right]^{-1}
$$


noncolluding or colluding eavesdroppers. The transmitter is equipped with multiple antennas and is able to simultaneously transmit an information signal to the intended receiver and artificial noise to confuse the eavesdroppers. We obtained a closedform expression for the ergodic secrecy capacity lower bound. We studied the optimal power allocation between transmission of the information signal and the artificial noise. In particular, equal power allocation was shown to be a near-optimal strategy in the case of noncolluding eavesdroppers. When the number of colluding eavesdroppers increases, more power should be used to generate artificial noise. We also derived an upper bound on the critical SNR, above which, the secrecy rate is positive, and this bound was shown to be tight at low SNR. When imperfect CSI was considered in the form of channel estimation errors, we found that it is wise to create more artificial noise to confuse the eavesdroppers than to increase the signal strength for the intended receiver. The results obtained in this paper provide various insights into the design and analysis of secure communication with multiantenna transmission.

\section{APPENDIX \\ IDENTITY FOR A SPECIAL CLASS OF GAUSS HYPERGEOMETRIC FUNCTION}

Here, we obtain a simplified expression for the Gauss hypergeometric function in the form of ${ }_{2} F_{1}(1,1 ; N+1 ; x)$ or ${ }_{2} F_{1}(N, N ; N+1 ; x)$ for the integer $N \geq 1$. From [28], we know that these two forms of the Gauss hypergeometric function are related to each other by

$$
{ }_{2} F_{1}(1,1 ; N+1 ; x)=(1-x)^{N-1}{ }_{2} F_{1}(N, N ; N+1 ; x) .
$$

In addition, we know from [28] that

$$
\frac{d^{N-1}}{d x^{N-1}}{ }_{2} F_{1}(1,1 ; 2 ; x)=\frac{(1)_{N-1}(1)_{N-1}}{(2)_{N-1}}{ }_{2} F_{1}(N, N ; N+1 ; x)
$$

where $(a)_{b}$ is the rising factorial. Therefore, we have

$$
\begin{aligned}
{ }_{2} F_{1} & (N, N ; N+1 ; x) \\
& =\frac{(2)_{N-1}}{(1)_{N-1}(1)_{N-1}} \frac{d^{N-1}}{d x^{N-1}}{ }_{2} F_{1}(1,1 ; 2 ; x) \\
& =-\frac{N}{(N-1) !} \sum_{l=0}^{N-1}\left(\begin{array}{c}
N-1 \\
l
\end{array}\right) \frac{d^{l}}{d x^{l}} \ln (1-x) \frac{d^{N-1-l}}{d x^{N-1-l}} x^{-1}
\end{aligned}
$$

where we have used the identity ${ }_{2} F_{1}(1,1 ; 2 ; x)=-\ln (1-$ $x) / x$ from [28]. It is easy to show that

$$
\begin{aligned}
\frac{d^{k}}{d x^{k}} \ln (1-x) & =-\frac{d^{k-1}}{d x^{k-1}}(1-x)^{-1} \\
& =-\frac{(k-1) !}{(1-x)^{k}}, \quad k=1,2,3, \ldots \\
\frac{d^{k}}{d x^{k}} z^{-1} & =\frac{(-1)^{k} k !}{x^{k+1}}, \quad k=0,1,2,3, \ldots
\end{aligned}
$$

Substituting the above expressions for the derivatives into (44), we obtain an identity expression as

$$
\begin{aligned}
{ }_{2} F_{1}(N, N ; N+1 ; x) \\
=-\frac{N}{(N-1) !} \\
\quad \times\left(\ln (1-x) \frac{(-1)^{N-1}(N-1) !}{z^{N}}\right. \\
\left.\quad-q \sum_{l=1}^{N-1} \frac{(N-1) !}{l !(N-1-l) !} \frac{(l-1) !}{(1-x)^{l}} \frac{(-1)^{N-1-l}(N-1-l) !}{x^{N-l}}\right) \\
=\frac{(-1)^{N} N}{x^{N}}\left(\ln (1-x)-\sum_{l=1}^{N-1} \frac{1}{l} \frac{x^{l}}{(x-1)^{l}}\right) .
\end{aligned}
$$

Using (43), we also have

$$
\begin{aligned}
{ }_{2} F_{1} & (1,1 ; N+1 ; x) \\
& =\frac{(-1)^{N} N(1-x)^{N-1}}{x^{N}}\left(\ln (1-x)-\sum_{l=1}^{N-1} \frac{1}{l} \frac{x^{l}}{(x-1)^{l}}\right) .
\end{aligned}
$$

\section{ACKNOWLEDGMENT}

The authors would like to thank Dr. P. Sadeghi for useful discussions.

\section{REFERENCES}

[1] A. Wyner, "The wire-tap channel," Bell Syst. Tech. J., vol. 54, no. 8, pp. $1355-1387$, Oct. 1975

[2] I. Csiszár and J. Körner, "Broadcast channels with confidential messages," IEEE Trans. Inf. Theory, vol. IT-24, no. 3, pp. 339-348, May 1978.

[3] Z. Li, W. Trappe, and R. Yates, "Secret communication via multiantenna transmission," in Proc. 41st CISS, Baltimore, MD, Mar. 2007, pp. $905-910$.

[4] S. Shafiee and S. Ulukus, "Achievable rates in Gaussian MISO channels with secrecy constraints," in Proc. IEEE ISIT, Nice, France, Jun. 2007, pp. 2466-2470.

[5] A. Khisti and G. W. Wornell, "Secure transmission with multiple antennas: The MIMOME channel," IEEE Trans. Inf. Theory, to be published. [Online]. Available: http://allegro.mit.edu/pubs/posted/journal/2008khisti-wornell-it.pdf

[6] F. Oggier and B. Hassibi, "The secrecy capacity of the MIMO wiretap channel," in Proc. IEEE ISIT, Toronto, ON, Canada, Jul. 2008, pp. 524-528.

[7] P. Parada and R. Blahut, "Secrecy capacity of SIMO and slow fading channels," in Proc. IEEE ISIT, Adelaide, Australia, Sep. 2005, pp. 2152-2155.

[8] J. Barros and M. R. D. Rodrigues, "Secrecy capacity of wireless channels," in Proc. IEEE ISIT, Seattle, WA, Jul. 2006, pp. 356-360.

[9] Y. Liang, H. V. Poor, and S. Shamai, "Secure communication over fading channels," IEEE Trans. Inf. Theory, vol. 54, no. 6, pp. 2470-2492, Jun. 2008.

[10] P. K. Gopala, L. Lai, and H. E. Gamal, "On the secrecy capacity of fading channels," IEEE Trans. Inf. Theory, vol. 54, no. 10, pp. 4687-4698, Oct. 2008.

[11] A. Khisti and G. W. Wornell, "Secure transmission with multiple antennas: The MISOME channel," IEEE Trans. Inf. Theory, vol. 56, no. 7, pp. 3088-3104, Jul. 2010.

[12] M. L. Jorgensen, B. R. Yanakiev, F. E. Kirkelund, P. Popovski, H. Yomo, and T. Larsen, "Shout to secure: Physical-layer wireless security with known interference," in Proc. IEEE GLOBECOM, Washington, DC, Nov. 2007, pp. 33-38. 
[13] O. Simeone and P. Popovski, "Secure communications via cooperating base stations," IEEE Commun. Lett., vol. 12, no. 3, pp. 188-190, Mar. 2008.

[14] X. Tang, R. Liu, P. Spasojevic, and H. V. Poor, "Interference-assisted secret communication," in Proc. IEEE ITW, Porto, Portugal, May 2008, pp. $164-168$.

[15] L. Lai and H. E. Gamal, "The relay-eavesdropper channel: Cooperation for secrecy," IEEE Trans. Inf. Theory, vol. 54, no. 9, pp. 4005-4019, Sep. 2008

[16] L. Dong, Z. Han, A. P. Petropulu, and H. V. Poor, "Secure wireless communications via cooperation," in Proc. 46th Annu. Allerton Conf. Commun., Control, Comput., Monticello, IL, Sep. 2008, pp. 1132-1138.

[17] L. Dong, Z. Han, A. P. Petropulu, and H. V. Poor, "Amplify-and-forward based cooperation for secure wireless communications," in Proc. IEEE ICASSP, Taipei, Taiwan, Apr. 2009, pp. 2613-2616.

[18] E. Tekin and A. Yener, "The general Gaussian multiple-access and twoway channels: Achievable rates and cooperative jamming," IEEE Trans. Inf. Theory, vol. 54, no. 6, pp. 2735-2751, Jun. 2008.

[19] E. Ekrem and S. Ulukus, "Secrecy in cooperative relay broadcast channels," IEEE Trans. Inf. Theory, submitted for publication. [Online]. Available: http://arxiv.org/abs/0811.1317

[20] E. Ekrem and S. Ulukus, "Cooperative secrecy in wireless communications," in Securing Wireless Communications at the Physical Layer, R. Liu and W. Trappe, Eds. New York: Springer-Verlag, 2009.

[21] R. Negi and S. Goel, "Secret communications using artificial noise," in Proc. IEEE VTC, Dallas, TX, Sep. 2005, pp. 1906-1910.

[22] S. Goel and R. Negi, "Guaranteeing secrecy using artificial noise," IEEE Trans. Wireless Commun., vol. 7, no. 6, pp. 2180-2189, Jun. 2008.

[23] A. L. Swindlehurst, "Fixed SINR solutions for the MIMO wiretap channel," in Proc. IEEE ICASSP, Taipei, Taiwan, Apr. 2009, pp. 2437-2440.

[24] S. Verdú, "Spectral efficiency in the wideband regime," IEEE Trans. Inf. Theory, vol. 48, no. 6, pp. 1319-1343, Jun. 2002.

[25] G. Alfano, A. Lozano, A. M. Tulino, and S. Verdú, "Mutual information and eigenvalue distribution of MIMO Ricean channels," in Proc. ISITA, Parma, Italy, Oct. 2004

[26] H. Gao, P. J. Smith, and M. V. Clark, "Theoretical reliability of MMSE linear diversity combining in Rayleigh-fading additive interference channels," IEEE Trans. Commun., vol. 46, no. 5, pp. 666-672, May 1998.

[27] I. S. Gradshteyn and I. M. Ryzhik, Tables of Integrals, Series, and Products, 7th ed. New York: Academic, 2007.

[28] M. Abramowitz and I. A. Stegun, Handbook of Mathematical Functions With Formulaes, Graphs, and Mathematical Tables. New York: Dover, 1974.

[29] T. Yoo and A. Goldsmith, "Capacity and power allocation for fading MIMO channels with channel estimation error," IEEE Trans. Inf. Theory, vol. 52, no. 5, pp. 2203-2214, May 2006.

[30] B. D. O. Anderson and J. B. Moore, Optimal Filtering. New York: Dover, 1979.

[31] B. Hassibi and B. M. Hochwald, "How much training is needed in multiple-antenna wireless links?" IEEE Trans. Inf. Theory, vol. 49, no. 4, pp. 951-963, Apr. 2003.

[32] I. E. Telatar, "Capacity of multi-antenna Gaussian channels," Eur. Trans. Telecommun., vol. 10, no. 6, pp. 585-595, Nov. 1999.

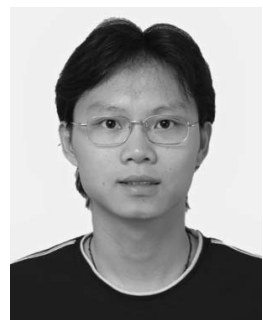

security.

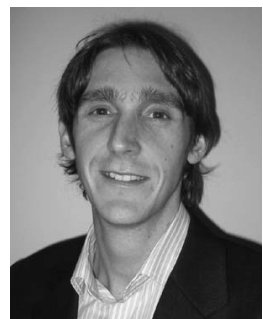

Matthew R. McKay (S'03-M'07) received the combined B.E. degree in electrical engineering and B.I.T. degree in computer science from the Queensland University of Technology, Brisbane, Australia, in 2002 and the Ph.D. degree in electrica engineering from the University of Sydney, Sydney, Australia, in 2006.

He was a Research Scientist with the Commonwealth Science and Industrial Research Organization, Sydney, Australia, prior to joining the faculty at the Hong Kong University of Science and Technology (HKUST), Kowloon, Hong Kong, in 2007, where he is currently an Assistant Professor. He is also a member of the Center for Wireless Information Technology, HKUST. His research interests include communications and signal processing, in particular, the analysis and design of multiple-input-multipleoutput systems, random matrix theory, information theory, and wireless ad hoc and sensor networks

Dr. McKay received a Best Student Paper Award at the 2006 IEEE International Conference on Acoustics, Speech, and Signal Processing and jointly received the Best Student Paper Award at the 2006 IEEE Vehicular Technology Society Spring Conference. He was also awarded the University Medal upon graduating from the Queensland University of Technology. 